\title{
Quality of Service and Quality of Experience Correlations in a Location-Based Mobile Multiplayer Role-Playing Game
}

\author{
Maarten Wijnants ${ }^{1}$, Wouter Vanmontfort ${ }^{1}$, Jeroen Dierckx ${ }^{1}$, Peter Quax ${ }^{1}$, \\ Wim Lamotte ${ }^{1}$, Katrien De Moor ${ }^{2}$, and Jeroen Vanattenhoven ${ }^{3}$ \\ ${ }^{1}$ Hasselt University - tUL - IBBT - Expertise Centre for Digital Media \\ Wetenschapspark 2, 3590 Diepenbeek, Belgium \\ firstname. lastname@uhasselt. be \\ ${ }^{2}$ IBBT-MICT - Department of Communication Sciences - Ghent University \\ Korte Meer 7-11, 9000 Ghent, Belgium \\ KatrienR. DeMoor@ugent. be \\ ${ }^{3}$ Centre for User Experience Research - KULeuven - IBBT \\ Parkstraat 45 bus 3605, 3000 Leuven, Belgium \\ jeroen.vanat tenhovenasoc. kuleuven. be
}

\begin{abstract}
The well-established Quality of Service and the relatively recently emerged Quality of Experience research topics are unquestionably linked, yet to date little effort has been invested in the study of their precise correlation and mutual reciprocity. This paper examines the impact of three representative QoSrelated parameters on user QoE in the particular context of location-based multiplayer games played on mobile devices. The choice for this research context is motivated by the rapidly expanding popularity of this application domain. Analysis of the quantitative and qualitative results from an empirical study involving 32 participants confirms that modifications in the performance of the investigated technical parameters does indeed hold implications for the quality of the users' experiences; at the same time, the results clarify the magnitude of these implications. Our findings are valuable as they provide practical insight in the QoS/QoE relationship and can hence aid software developers in delivering high-quality usage experiences to their customers.
\end{abstract}

Keywords: QoS, QoE, quantitative and qualitative user study, location-based mobile gaming, multiplayer, user experience.

\section{Introduction and Background}

Quality of Service (QoS) is a notion that has been around for quite some time and that has become a well-established concept in software and system engineering. It acts as a measure of technological performance and excellence which, in general, denotes the quality of systems, networks or applications. QoS is especially frequently used in the domain of computer networking, where it refers to the ability of the transportation network to guarantee a certain performance level, for instance in terms of throughput, maximal delay, average delay variation, bit error rate, and so on. In summary, although being a relatively extensive construct, QoS focuses exclusively on technical dimensions. As a direct consequence, it is objectively measurable. 
In contrast, Quality of Experience (QoE) has only fairly recently become an active research topic. Attention for QoE has emerged in the late 1990s but has since then increased exponentially. QoE can be considered the semantic variant of QoS since, broadly speaking, it denotes the overall experience that is witnessed by an end-user. Stated differently, it refers to a consumer's satisfaction when using a particular product or service. As such, it is clear that it is a humane and highly subjective metric that is hard or even impossible to quantify objectively. Compared to QoS, it is also much more multi-disciplinary since it might involve aspects from several nontechnological domains, including sociology, cognitive sciences, psychology, usability research, user interface design, context-aware computing, expectation modeling and market studies.

Given its broad scope, QoE definitions abound in the literature. Probably one of the most elaborate and versatile definitions is provided by $\mathrm{Wu}$ et al. in [10]. In this article, the term QoE will be wielded loosely to denote the experience or the satisfaction of the user, in this case while playing a multiplayer location-based mobile game that requires data communication via a transportation network.

The QoS and QoE concepts are irrefutably related. This is for instance manifested in the fact that, somewhat paradoxically perhaps, the majority of initial studies on user experience optimization was concentrated primarily on technical performance and QoS achievements. Even to date, a strong attention for technological factors remains explicit in user research. For example, Eberle et al. have developed a cross-layer methodology for trading off performance versus energy consumption in wireless communication environments, but they largely neglect actual user satisfaction in the process [1]. Soldani has investigated QoE in a telecommunications setting (i.e., for cellular phone users) and claims that QoE performance is defined by service accessibility, retainability, availability and integrity [8], which are all purely technical elements. As a final example, Vanhaverbeke et al. quantify user experience in HDTV video streaming setups exclusively as the number of Visible Distortions in Twelve hours (VDTs) and propose Forward Error Correction (FEC) and retransmission schemes as means to improve video quality and hence user satisfaction [9].

Unfortunately, the exact relationship between QoS and QoE to date remains largely uninvestigated and therefore unclear. Such information nonetheless seems very valuable for developers of (distributed) applications. For example, a decent understanding of the mutual impact of QoS and QoE dimensions as well as their interaction with each other is likely to allow software developers to optimize the experience of their users. This in turn might prove an important asset in Customer Relationship Management (CRM) in general, and in achieving customer loyalty in particular. The main contribution of this paper is that it unveils some of the correlations that exist between QoS and QoE by means of a user research methodology comprising both quantitative and qualitative components.

The field of mobile gaming was selected as context to conduct our research. This decision is motivated by the steadily expanding popularity of this application domain, which is for instance exemplified by the widespread penetration of handheld game consoles such as the Nintendo DS in the mainstream consumer market. Two remarks regarding the adopted research field are in place. It is first of all stressed that we focus our attention exclusively on distributed multiplayer games. Such games allow multiple individuals to share a single game environment and to interact with each 
other in this environment, be it in the form of partnership, competition or rivalry. To enable the interaction, multiplayer games typically rely on a transportation network to disseminate information among involved players. As a result, the operation and performance of the networking substrate might substantially influence user experience. Secondly, the scope of our research was further narrowed down to games which revolve around location determination. In such games, the position of players and/or objects in the physical world is tracked and plays a prominent role in the gameplay. Modern hardware that can be used for mobile gaming purposes (e.g., handheld consoles, smartphones) typically comes equipped with a number of peripherals (like a GPS module and/or a digital compass) that can be exploited to implement localization in outdoor environments. Alternatively, location determination can be achieved via triangulation of the device's wireless signal. Example locationbased games include Tourality (http://www.tourality.com/), an outdoor GPSbased multiplayer scavenger hunt game, and Pac-Manhattan, a large-scale urban game that recreates the 1980s video game sensation Pac-Man in the streets of New York City (http: / /www. pacmanhattan.com/).

In the just delineated gaming context, the effect of three concrete technologyrelated parameters on user experience was studied by means of hybrid quantitative and qualitative user research. This yielded a number of significant findings regarding the QoS/QoE relationship. These findings can function as high-level directives for developers of mobile location-based games (and potentially also other types of distributed mobile applications): if they are taken into account during design and implementation, the expected outcome is an improved QoE for the end-user. A secondary contribution of this article is hence that it transfers valuable knowledge concerning QoS and QoE interactions to developers of distributed mobile software.

The outline for the remainder of this article is as follows. Section 2 describes the location-based multiplayer game that was used to conduct our research. Next, section 3 overviews the technical parameters that were studied for their potential impact on player QoE. All facets of the performed user research, including practical considerations, employed methodology and result analysis, are grouped in section 4. Finally, section 5 presents our conclusions.

\section{Research Context: Location-Based Role-Playing Game}

The investigation of QoS and QoE correlations occurred by means of an in-house developed mobile location-based multiplayer game. The game stages a World War I (WW I) setting and was originally intended to promote WW I musea (and tourism in general) in Belgium. The game is played outdoors between two rivaling teams that respectively represent the Allied forces and the German army. It is a location-based game in the sense that the gameplay involves players walking around in the physical world while carrying a handheld device (currently a small Gigabyte T1000 netbook; in the future potentially a PDA or smartphone). Players' physical movement is tracked via a GPS receiver that is attached to the portable device and is distributed among the other participants, after which it is visualized on a satellite view of the outdoor environment [3]. 
Players can interact with both other participants and with the environment through QR tags, an ISO/IEC standardized 2D barcode specification [5]. In particular, each player carries such a (unique) QR code and there are also a number of codes scattered around the playing area, which each represent a virtual item. By photographing a QR code with the built-in camera of their portable device, players respectively start an interaction with the corresponding user or pick up the associated virtual item. This mode of interacting with virtual objects via $Q R$ tags can be considered a form of Augmented Reality (AR).

The game can be designated a Role-Playing Game (RPG) in that it enables players to take on different profiles. The selected role determines which actions the player can undertake in the game, what his/her objectives are and which virtual items he/she can gather in the real world via QR code scanning. The supported roles are the following:

Commander Does not enter the playing field but instead remains at a fixed indoor location (i.e., a stationary PC). The Commander has a global overview of the actions of his entire team. He also permanently sees the objects that are discovered by his outdoor team members and can instruct the players on the field (e.g., about the position of these objects).

Soldier Needs to search for weapons in the playing field and can use these weapons to engage players from the opposing team.

Spy Is responsible for acquiring intelligence items and is able to scan his surroundings to uncover the location of enemy players.

Medic Needs to collect medical supplies and can use these to heal injured teammates

(by scanning their QR code); can also revive killed players.

Figure 1 shows a screenshot of the game. Each player and discovered virtual object is represented by means of a marker on a top-down map of the real-life environment in which the game is played. As players relocate in the physical world, their marker is relocated accordingly. In contrast, item markers will never relocate in the course of a single gaming session. There are some restrictions on marker visualization: (i) only teammates are permanently visible on the map; (ii) members of the rivaling team become visible for a short period of time when they attack you (only applicable for an enemy Soldier) and when a Spy scans for their presence in his immediate vicinity; (iii) items only become visible on the map once they are discovered in the physical environment by a teammate; in addition, items are only displayed for a short amount of time on the virtual map for outdoor players, but remain permanently visible for the team's Commander and for the player that discovered the item.

The game includes inter-player communication facilities in the form of voice chat between teammates. This is implemented via a push-to-talk mechanism (see figure 1). Speech data (like all game-related data) is disseminated among participants via the $3 \mathrm{G}$ connection of the mobile device.

A gaming session lasts a predefined amount of time. The objective of the game is to accumulate as much points as possible during this time interval; the team with the highest aggregate score at the end of the session is the winner of the match. Players receive points for picking up virtual items and for the actions which they perform (e.g., a healing effort by a Medic). Different items and actions yield different revenue. 


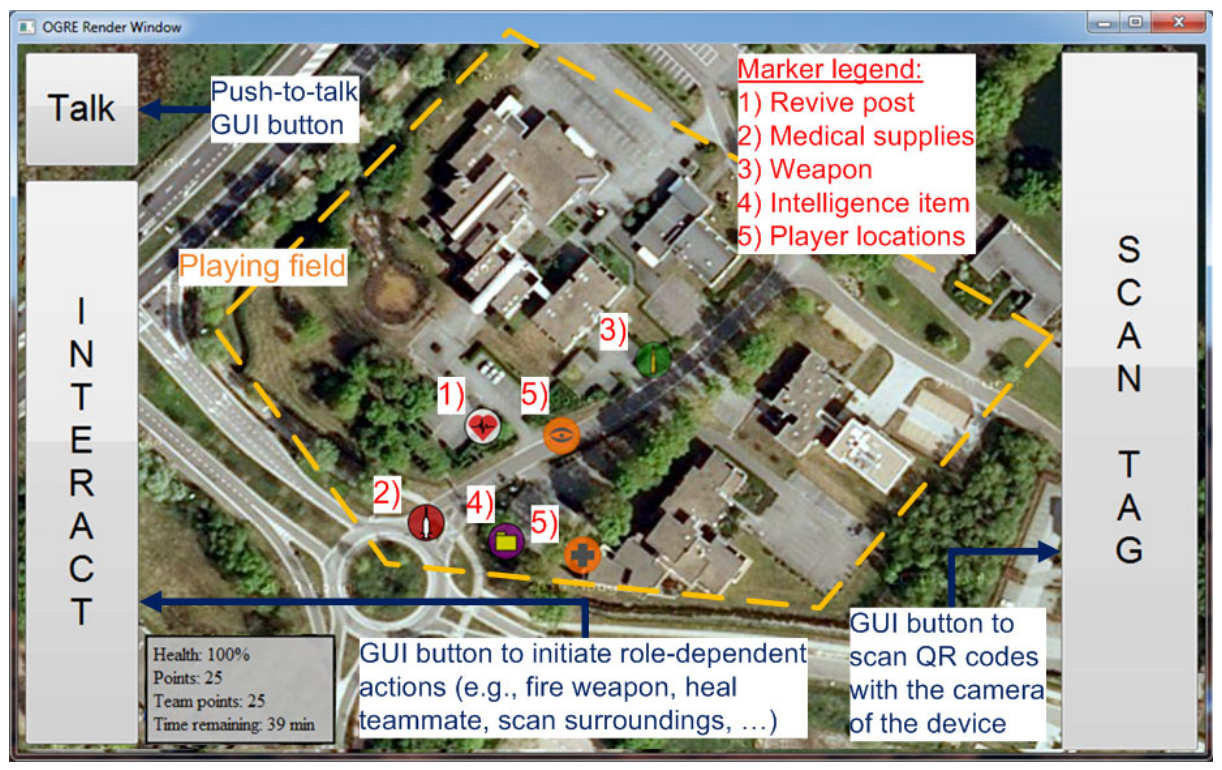

Fig. 1. The main screen of the game displays a satellite view of the real-life environment

\section{Investigated Technological Factors}

In the context of the just described mobile game, the influence of three QoS-related parameters on the QoE perceived by players was studied. Each parameter acted as a separate test condition and was hence investigated in isolation. It is hereby important to note that the test conditions themselves are representative (i.e., the simulated situations might very well occur in a real system), whereas the magnitudes used in each condition might somewhat be exaggerated. The choice for unrealistically high values is motivated by the fact that these were expected to elicit pronounced and unambiguous reactions from test subjects.

\subsection{Precision of Player Location Determination}

Localization techniques might not always be completely reliable. As an example, the accuracy of GPS-based solutions is affected by a number of factors, including atmospheric conditions (e.g., clouds) and natural barriers (e.g., buildings in an urban environment). It seemed interesting to determine the impact of such imprecisions on player QoE. We therefore developed a test condition in which the position of players on their team members' map was artificially and randomly varied in a 10 meter radius around the actual location. In practice, this resulted in a rapid bouncing motion, in a circular area, of the position of the player.

\subsection{Player Location Refresh Rate}

The rate at which player positions are disseminated forms an important design parameter for location-based games. A higher refresh rate yields more accurate 
positioning, but at the same time injects additional traffic in the transportation network. Stated differently, the location update pace allows for the trade-off between network load and data staleness to be controlled.

Under normal operation, player positions were updated multiple times per second in the game. A test condition was however implemented in which locations were only refreshed every 6 seconds. The outcome was a situation in which player visualizations discretely jumped from one position to the next at 6-second intervals (instead of producing smooth motion).

\subsection{Voice Communication Delay}

Communication and interaction in distributed applications is affected by the delay that is induced by the transportation network. Multiple factors can attribute to delay buildup, including congestion, failing links and bandwidth constraints. Regardless of the type of application, the International Telecommunication Union (ITU) recommends not to exceed a one-way latency of 400 milliseconds [6]. In this test condition, an additional voice delay of 2 seconds was artificially enforced on top of the latency that was implicitly introduced by the $3 \mathrm{G}$ network. As a result, fluid interpersonal communication was radically impeded because only 2 seconds after a sentence was uttered by a player, it was received by his team members.

\section{User Research}

\subsection{Pilot Testing}

Prior to the actual user studies, an iterative phase of pilot testing was organized in order to optimize the test procedure, to verify the robustness and stability of the game and to evaluate the implemented technical simulations. The pilot tests revealed that several users experienced problems with the QR tag scanning. Given the fact that it forms a cornerstone of the gameplay and is hence vital for making progress in the game, a tutorial application was developed to allow participants to master this operation. The pilot studies also resulted in a refinement of the user instructions and surveys in terms of clarity and layout.

\subsection{Practical Approach and Participant Demography}

Four separate user studies were organized with 8 test subjects each. In every study, the participants were evenly divided in 2 teams and each participant assumed a different role in his/her team (i.e., there was consistently one Commander, Soldier, Spy and Medic per team). The assignment of roles to players was randomized. The test subjects were beforehand deliberately not informed about the QoS manipulation and its resulting test conditions.

$12.5 \%$ of the test population were female. The average age of the participants was 29 years (Standard Deviation 9.04); the youngest was 16 years old, the oldest 57. More than $80 \%$ had played a video game during the past month. In terms of professional occupation, a great deal of the sample consisted of students (just over 40 $\%$ ) and employees (almost $50 \%$ ). 
The gaming sessions took place at a 7 hectare research and science park in Diepenbeek, Belgium (see figure 1). A total of 25 virtual objects (in the form of QR codes) were strategically dispersed in this area. These consisted of 3 revive posts, 6 medical supply items, 4 intelligence items and 12 weapons.

\subsection{Test Procedure and QoE Data Collection Methodology}

Every test session lasted approximately 2 hours and logically enveloped 3 consecutive phases. In the pre-usage phase, the participants were welcomed by the researchers and given instructions concerning the test procedure. Next, the objectives and mechanics of the location-based game were verbally outlined. During and after this short presentation, the test subjects were able to ask for further clarification. The participants were then requested to complete a questionnaire (on paper). This survey was intended to collect background information about the test subjects in terms of basic socio-demographical characteristics, as well as to establish previous experiences and expectations concerning digital games in general, and the WW I game in particular. To conclude the pre-usage stage, all users needed to complete the QR tag scanning tutorial to become acquainted with this particular gameplay element (see section 4.1).

Subsequently, the during-usage phase commenced, which spanned 40 minutes and was conceptually divided in 4 distinct intervals of equal length. Three intervals were each exclusively devoted to one of the test conditions described in section 3. Stated differently, in each of these time periods, one of the test conditions was separately activated in order to examine the influence of the corresponding QoS parameter on the test subjects' quality perception of the experience. The fourth interval on the other hand represented a period of "perfect" or "normal" gameplay in which no artificial technical impairments or manipulations were applied. This period hence acted as the reference scenario with which the player QoE findings from the other three intervals could be compared. The assignment of test conditions to time intervals was randomized, which yielded the following order: player location distortion, voice communication delay, normal gameplay and reduced player location update rate.

In the course of the playing session, 4 identical in-game questionnaires needed to be filled in by the players (via an electronic form displayed on their portable device). These surveys were evoked at the same moment for every test user, namely at the end of each of the conceptual intervals of the session. It had been explained in the preusage briefing that these questionnaires would pop up and that the test subjects needed to complete and submit them before they would be able to continue the game.

The in-game inquiries were included in the data compilation methodology with the purpose of acquiring detailed information related to relevant QoE aspects in each of the test conditions. The downside of this strategy is that it (briefly) interrupts the gaming experience. On the positive side however, it enables sampling of the players' thoughts, emotions and behaviors (in this case, concerning the individual test conditions) at the very moment (or at least immediately after) they were actually experienced. Compared to an exclusively post-usage probing method (i.e., after all conditions have finished), an in-situ approach substantially reduces the risk of the recall bias effect and therefore is likely to yield more accurate and reliable assessments [2]. In this study, we particularly emphasized experience dimensions 
related to the considered technical factors (see section 3) and to the general concepts of absorption, fun, effort, frustration and expectations. The in-game survey comprised the following 11 statements that were each measured on a five-point Likert scale [7] (ranging from "1: Totally disagree" to "5: Totally agree"):

1. I feel totally absorbed in my role in the game

2. With the information that I am receiving about the position of the other players, I am able to localize them without a problem

3. I am having fun while playing the game

4. I have to put a lot of effort in the game

5. The kind of information that I am getting about the location of objects, allows me to localize these objects without a problem

6. I expected more from the game

7. While communicating with team members I experienced technical problems

8. I feel frustrated

9. The location of the other players is updated sufficiently

10. I am able to communicate smoothly with my team members

11. The location of objects is updated sufficiently

Immediately after the actual gaming session, the participants were again convoked for the post-gaming stage of the study. The test subjects were presented a second paperbased questionnaire in which they were inquired about their overall experiences, feelings and thoughts after having played the game. Finally, each user study was wrapped up with a group conversation in which all participants were invited to share their experiences, to elaborate on QoE-related aspects reported on in the questionnaires and to reflect on QoE factors that were not covered by any of the questionnaires.

\subsection{Quantitative and Qualitative Findings}

The pre-usage questionnaire responses indicated that virtually every participant had high expectations concerning the game: a very large majority was curious to try the game $(96.8 \%)$, appreciated the possibility to communicate with other players while playing (93.4\%), liked the fact that they could assume different roles $(93.5 \%)$ and expected that the WW I game would be fun to play $(96.7 \%)$. On the other hand, only a minority had the impression that there was a story in the game $(33.3 \%)$ and that story seemed fascinating $(25.8 \%)$. Yet, few test users were worried that they would be bored quickly or that it would be difficult for them to get absorbed in the game.

The 11 QoE-related statements that were sampled during the gaming sessions were also covered by both the pre- and post-usage surveys. The pre-usage responses indicated that test subjects attached most importance to the 'having fun' (Mean=4.58), 'not having technical problems while communicating with team members' $(M=4.42)$ and 'being able to communicate smoothly with team members' $(M=4.23)$ aspects before they had actually played the game. Analysis of the post-usage data resulted in the same top 3 list. 


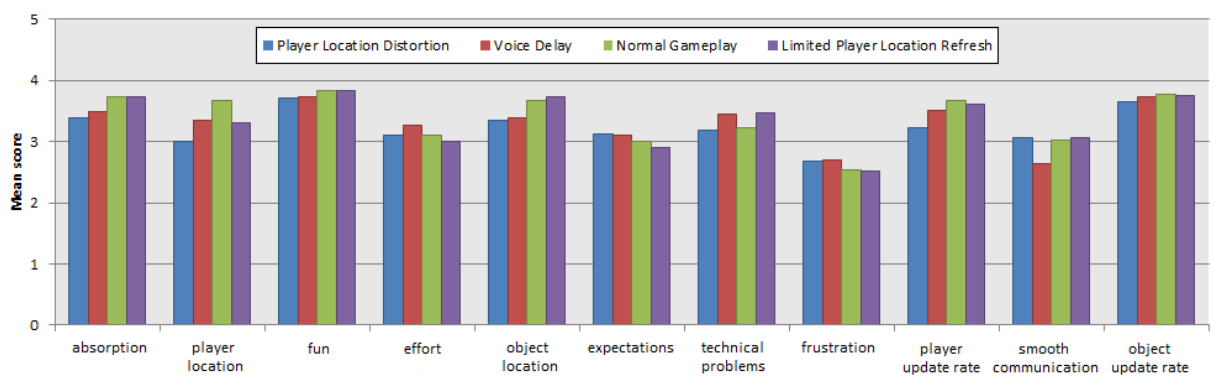

Fig. 2. Mean scores for the in-game questionnaire statements per test condition

Figure 2 compares the mean scores for the statements from the in-game questionnaires for each of the four conceptual test intervals. Before zooming in on the individual intervals, it is worth discussing the global characteristics of the results regarding the non-instrumental dimensions. It can be witnessed from the chart that users felt least 'absorbed' in their role in the first condition and that this feeling monotonously increased as the gaming session progressed. A similar yet less pronounced evolution applies to the 'fun' aspect. A possible explanation for these trends might be that the test subjects needed to get used to the game, its rules, mechanics and features before they were able to become immersed and to have fun while playing. Another interesting high-level observation is that the expectations were adjusted in the course of the gaming session, since the average scores for 'expected more from it' decrease with every condition. It was known from the pre-usage phase that the participants' expectations were high; these figures support the idea that people constantly adapt their expectations according to the context and circumstances.

In interval 1 , the accuracy of the location-related information concerning other players as well as objects was rated lower than in the other test conditions. Furthermore, the artificially enforced player location distortion appears to have influenced the users' perception of location update rate (of both players and objects), since the scores in this respect were also minimal during this interval.

The participants felt most 'frustrated' during the second test condition, in which the voice communication was intentionally delayed. Moreover, they indicated that this interval required the most 'effort' from them. Finally, the rating for 'experiencing technical problems in the communication with team members' was considerably higher than in conditions 1 and 3. This difference can also be observed in statement 10 ('I am able to communicate smoothly with my team members'), for which the average score in condition 2 is clearly bottommost.

In the third interval, which was not artificially manipulated in any way, we observed the highest degree of 'absorption' in the played role and also a maximal score for 'fun'. The same goes for the accuracy of the player location information, as well as for the position refresh rate of both players and objects.

In the last condition, player location updates were disseminated less frequently. The figures however disclose that this technical manipulation did not have a notable influence on the monitored QoE dimensions (this claim is also supported by the qualitative data from the post-gaming stage, see later on). The 'frustration' of the test subjects was the lowest in this condition, as was their estimation of the amount of 
'effort' that they had to invest in the game. Similarly, in terms of pleasure or 'fun', a high average score was reported. An important comment in this respect concerns the fact that the surface area of the playing field was relatively small. Whereas at the beginning of the session the players from both teams typically were actively dispersing and exploring the environment, they were near the end rather centrally clustered because the entire playing area had already been discovered. As a result, they had to rely less on the information displayed on their device (since there was more direct visual contact between players), which might account for these findings.

Although the differences between the four conditions are in some cases subtle and hardly explicit, they do corroborate that our study succeeded in relating modifications in technical circumstances to the users' (subjective) experiences.

In the post-usage phase, the participants were asked to indicate their level of frustration on a scale from 1 to 10 . The mean score was $4.17(\mathrm{SD}=2.52)$. Analogous to the findings from the in-game surveys, the group discussions and post-gaming questionnaire responses revealed that the manipulated factors during the first and second playing condition were experienced as sources of frustration. One participant remarked: "We noticed the [audio] delay because we were standing next to each other, but not everyone noticed". Another user indicated that the meddling with the GPS update rate (interval 4) did not have that much impact on the gameplay: "The GPS update rate wasn't really a problem; most of the time they weren't running, so you could easily track the other players". One test subject formulated the consequences of the distortion of the GPS location for the Commander as follows: "As Commander I expect to know the precise location of my team; the lag on the positions made this impossible". The same aspect is mentioned by another participant: "My instructions didn't arrive well, and the position of the players wasn't very accurate". For one player, the artificial impairment of the communication had a severe impact on his state of mind: "I had a lot of problems with the communication and felt aimless at times". Finally, it is important to note that the post-gaming data uncovered that the technical manipulations were not the only sources of frustration. The scanning of the tags was considered cumbersome and was mentioned by several participants as a significant source of frustration. Also, the Commander role was found to be the least challenging because of its stationary indoor location. Finally, the portable device was judged a bit heavy and ponderous for playing the game.

Finally, it was also investigated whether the role that participants played had an influence on their experience. In this respect, a one-way ANOVA [4] was computed using player role as a factor and the scores on the in-game survey statements as dependents (see figure 3). This analysis disclosed that there were significant differences (at .05 level) between the various roles for a total of 5 aspects. A post-hoc Scheffe test was conducted to locate these differences. At the level of 'absorption' in their role, Soldiers felt notably more immersed than Commanders. Moreover, the Soldiers had more 'fun' compared to the Medic and Commander roles. The same goes for the 'effort' that the players put in the game: this value was significantly higher for the Soldier than for the other roles, and especially the Commander. In terms of 'expectations', the opposite is true: the expectations of the Commanders were significantly higher than those of the Soldier players. Finally, Commanders were considerably less satisfied with the player location update rate. This observation 
also emerged in the post-usage group discussions: since the Commander was not located in the field, precise player position information was more crucial for him than for the other types of players. In addition, due to the characteristics of their role, Commanders could fix their attention to the screen, whereas outdoor players also had to take notice of the physical environment; it is therefore to expect that discrepancies in teammates' on-screen locations were noticed sooner and more apparently by the Commander.

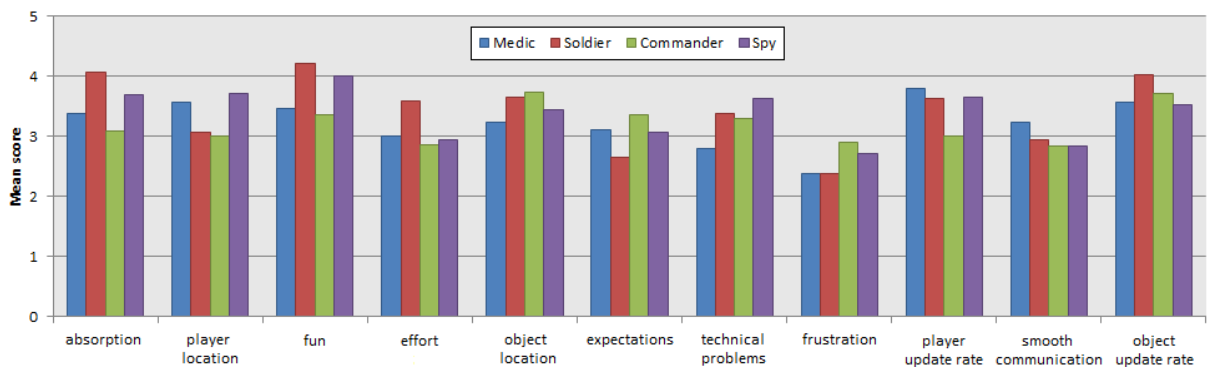

Fig. 3. Mean scores for the in-game questionnaire statements per player role

\section{Conclusions}

The main objective of the presented study was to explore the dependencies between the QoS and QoE concepts in the context of a mobile, location-based role-playing game. Two high-level conclusions can be drawn from our findings. First of all, it has been established that modifications in three specific technical parameters did indeed hold implications for the quality of the users' experiences. In particular, during the test condition in which no artificial QoS impairments were applied, the highest average scores for the feelings of 'absorption' and 'fun' were recorded. Secondly, for some sub-dimensions, the player reported QoE has been found to diverge depending on the assumed role. It has for instance been determined that Soldiers had significantly more 'fun' than Medic and Commander players and that they also felt most 'absorbed' in their role. For both global conclusions, the qualitative data nicely complemented the quantitative findings and helped to understand the identified quantitative disparities between the distinct test conditions and player roles.

Besides the general conclusions, the field study has also produced a number of interesting lower-level, specific findings that illustrate the extent of the impact of QoS performance on user experience. A notable example is that severe latency in the voice communication has been proven to complicate the coordination of players' actions, which made them feel less in control and less 'absorbed' in the game; moreover, this test condition yielded the highest mean ratings for 'frustration' and required 'effort'. The value of such fine-grained information is that it might assist software developers in their ever-present quest for providing optimal user experiences, since it informs them how best to cope with irregularities in the quality of the three investigated instrumental parameters. 
Finally, a prominent result of the presented research is that the feelings of 'absorption' and 'fun' monotonously improved in the course of the playing session and that test subjects constantly adjusted their expectations of the game. This implies that, despite the artificially induced technical imperfections, the overall evaluation of the participants was positive in the sense that they found it a fun and engaging experience. This finding confirms that QoE exceeds QoS in terms of scope, since it corroborates that overall user experience is not merely determined by technical parameters (i.e., lower technical performance did not automatically result in an unacceptable player experience). As such, our study has illustrated the importance and potential influence of non-instrumental aspects on QoE and opens opportunities for future research in this regard.

Acknowledgments. This research is part of the IBBT Gr@sp project, funded by the Flemish government. Part of this research is also funded by the EFRD.

\section{References}

1. Eberle, W., Bougard, B., Pollin, S., Catthoor, F.: From Myth to Methodology: CrossLayer Design for Energy-Efficient Wireless Communication. In: Proceedings of DAC 2005, Anaheim, CA, USA, pp. 303-308 (2005)

2. Froehlich, J., Chen, M.Y., Consolvo, S., Harrison, B., Landay, J.A.: MyExperience: A System for In Situ Tracing and Capturing of User Feedback on Mobile Phones. In: Proceedings of MobiSys 2007, San Juan, Puerto Rico, pp. 57-70 (June 2007)

3. Google Code: Google Maps API, http://code.google.com/apis/maps/

4. Huizingh, E.: Applied Statistics with SPSS. SAGE Publications Ltd., Thousand Oaks (2007)

5. ISO/IEC 18004:2006: Information Technology - Automatic Identification and Data Capture Techniques - QR Code 2005 Bar Code Symbology Specification (August 2006), http: / /www.iso.org/iso/catalogue_detail.htm? csnumber $=43655$

6. ITU-T G.114: 114: One-way Transmission Time (May 2003), http://www.cs.columbia.edu/ andreaf/new/documents / other/T-REC-G.114-200305.pdf

7. Likert, R.: A Technique for the Measurement of Attitudes. Archives of Psychology 22(140), 1-55 (1932)

8. Soldani, D.: Means and Methods for Collecting and Analyzing QoE Measurements in Wireless Networks. In: Proceedings of WoWMoM, Buffalo, NY, USA, pp. 531-535 (June 2006)

9. Vanhaverbeke, F., Moeneclaey, M., Laevens, K., Degrande, N., De Vleeschauwer, D.: Video Quality Protection Strategies for HDTV in the Presence of Buffer Overflow. In: Proceedings of ICN 2009, Cancun, Mexico, pp. 342-346 (March 2009)

10. Wu, W., Arefin, A., Rivas, R., Nahrstedt, K., Sheppard, R., Yang, Z.: Quality of Experience in Distributed Interactive Multimedia Environments: Toward a Theoretical Framework. In: Proceedings of ACM MULTIMEDIA 2009, Beijing, China, pp. 481-490 (October 2009) 